\title{
Regularization, renormalization and "peratization" in effective field theory for two nucleons
}

\author{
E. Epelbaum ${ }^{1,2}$ and J. Gegelia ${ }^{3,4}$ \\ ${ }^{1}$ Forschungszentrum Jülich, Institut für Kernphysik (Theorie) and \\ Jülich Center for Hadron Physics, \\ D-52425 Jülich, Germany \\ ${ }^{2}$ Helmholtz-Institut für Strahlen- und Kernphysik (Theorie) and \\ Bethe Center for Theoretical Physics, Universität Bonn, \\ D-53115 Bonn, Germany \\ ${ }^{3}$ Institut für Kernphysik, Johannes Gutenberg-Universität, \\ D-55099 Mainz, Germany \\ ${ }^{4}$ High Energy Physics Institute, Tbilisi State University, \\ 0186 Tbilisi, Georgia
}

\begin{abstract}
We discuss conceptual aspects of renormalization in the context of effective field theories for the two-nucleon system. It is shown that, contrary to widespread belief, renormalization scheme dependence of the scattering amplitude can only be eliminated up to the order the calculations are performed. We further consider an effective theory for an exactly solvable quantum mechanical model which possesses a long- and short-range interaction to simulate pionful effective field theory. We discuss the meaning of low-energy theorems in this model and demonstrate their validity in calculations with a finite cutoff $\Lambda$ as long as it is chosen of the order of the hard scale in the problem. Removing the cutoff by taking the limit $\Lambda \rightarrow \infty$ yields a finite result for the scattering amplitude but violates the low-energy theorems and is, therefore, not compatible with the effective field theory framework.
\end{abstract}

PACS numbers: 11.10.Gh,12.39.Fe,13.75.Cs 


\section{INTRODUCTION}

Almost two decades ago Weinberg proposed a way to extend baryon chiral perturbation theory to few-nucleon systems [1, 2]. These seminal papers triggered an intensive research activity starting with the pioneering work of Ref. [3]. In this approach, chiral perturbation theory is applied to the effective potential, defined as the sum of all possible $N$-nucleon irreducible diagrams, rather than to the scattering amplitude. The amplitude is then generated by solving the corresponding dynamical equation such as the Lippmann-Schwinger (LS) equation in the two-nucleon sector. For recent reviews and references the reader is referred to Refs. [4, 5, 6].

While phenomenologically successful, the consistency of Weinberg's approach was questioned by several authors. The nucleon-nucleon (NN) potential in this formalism is nonrenormalizable in the traditional sense, i.e. iterations of the LS equation generate divergent terms with structures which are not included in the original potential. For example, the leading-order (LO) NN potential is given by derivative-less contact interactions contributing only to S-waves and the one-pion exchange (OPE) term whose spin-triplet part behaves at short distances as $1 / r^{3}$ and, therefore, generates divergences also in higher partial waves. Consequently, renormalization of the solution of the LS equation requires inclusion of contributions of infinitely many higher-order short-range operators in the potential (counterterms). The freedom in the choice of the finite parts of counterterms is compensated by the running of the corresponding renormalized coupling constants. It has been argued [7] that the coefficients in front of the divergent parts of the counterterms contributing at a given order set the scale of the corresponding renormalized couplings. As a consequence, even if these couplings were natural at some value of the renormalization scale, they would become unnaturally large for slightly different values of this parameter. This problem, also treated non-perturbatively, is what is usually referred to as inconsistency of Weinberg's approach, see also Ref. [8] for a related discussion.

An alternative power counting scheme has been proposed by Kaplan, Savage and Wise (KSW) [7, 9, 10, 11], in which the troublesome OPE contribution to the potential is shifted from LO to next-to-leading order (NLO). The LO dynamical equation becomes renormalizable, both perturbatively and non-perturbatively, i.e. all divergences can be absorbed into redefinition of low-energy constants (LECs) entering the potential. Moreover, the LO equation is exactly solvable and dimensional regularization can be applied. All corrections are treated perturbatively which guarantees that all divergences are absorbed into redefinition of parameters entering at a given order. Unfortunately the resulting perturbative expansion for the scattering amplitude was found not to converge for nucleon momenta of the order of the pion mass at least in certain spin-triplet channels [12], see however, Ref. [13] for a new formulation which is claimed to yield a convergent expansion. The reason for the breakdown of the KSW expansion was attributed to the perturbative treatment of the pion-exchange contributions [14, 15, 16, 17, 18]. This appears to be in line with phenomenological successes of Weinberg's approach which treats pion exchange contributions nonperturbatively. Indeed, the most advanced analyses of the NN system at next-to-next-to-next-to-leading order in the Weinberg's power counting scheme demonstrate the ability to accurately describe NN

scattering data up to center-of-mass momenta at least of the order $\sim 2 M_{\pi}[19$, , 20]. It is important to emphasize that these studies are carried out within the cutoff EFT along the lines of Lepage [21, 22, 23] who argued that the cutoff parameter in such calculations should be taken of the order of the relevant hard large scale such as e.g. the mass of the $\rho$ meson, 
see also Refs. [20, 24, 25, 26, 27, 28].

The fairly narrow range of cutoffs $\Lambda=450 \ldots 600 \mathrm{MeV}$ used in Refs. [19, 20] was criticized by Nogga et al. [29] who considered low NN partial waves based on the OPE potential and contact interactions employing a much larger range of cutoffs with $\Lambda<4 \mathrm{GeV}$. They found that higher-order counterterms have to be promoted to LO in the ${ }^{3} P_{0},{ }^{3} P_{2^{-}}{ }^{3} F_{2}$ and, possibly, the ${ }^{3} D_{2}$ channel in order to stabilize the amplitude in the employed cutoff range. The authors of Ref. [29] conjecture that the "mixture of perturbative treatment of higher partial waves, resummation of lower partial waves, and promotion of a finite number of counterterms is the most consistent approach" to chiral effective field theory (EFT) in the two-nucleon sector, see however, Ref. [28] for criticism. The possibility of a perturbative treatment of two- and more-pion exchange corrections to the potential was explored using renormalization-group methods [30, 31, 32]. Finally, the consequences of completely removing the cutoff $\Lambda$ by taking the limit $\Lambda \rightarrow \infty$ in the LS equation based on the NN potentials at various orders in chiral EFT are also being explored by several groups [33, 34, 35, 36, 37, 38, 39, 40, 41, 42, 43, 44, 45].

The purpose of this paper is to clarify some conceptual issues related to renormalization in the context of EFT for the two-nucleon system. First, we discuss renormalization scheme dependence of the scattering amplitude in the KSW and Weinberg's approaches. Contrary to widespread belief, we show that renormalization scheme independence in the KSW framework is only achievable up to the order to which the calculations are performed. From this point of view, the KSW framework does not offer any conceptual advantage over the Weinberg's approach. Secondly, we regard cutoff EFT and explore the consequences of completely removing (or taking very large values of) the cutoff. To that aim, we construct effective theory for an exactly solvable quantum mechanical model with long- $\left(r_{l} \sim m_{l}^{-1}\right)$ and short-range $\left(r_{s} \sim m_{s}^{-1} \ll m_{l}^{-1}\right)$ interactions of a separable type valid for momenta of the order $k \sim m_{l}$. This can be viewed as a toy-model for pionful EFT. We explain the meaning of low-energy theorems in this model using the KSW-like framework with subtractive renormalization and demonstrate their validity in the Weinberg-like approach with a finite cutoff $\Lambda$ as long as it is chosen of the order $\Lambda \sim m_{s}$. Next, it is shown that taking the limit $\Lambda \rightarrow \infty$ yields a finite result for the amplitude but leads to breakdown of low-energy theorems. This procedure is, therefore, not compatible with the EFT framework. We argue that $\Lambda$ should not be taken (considerably) larger than the short-range scale $m_{s}$ in the problem.

Our paper is organized as follows. In section II we consider renormalization scheme dependence in the KSW and Weinberg's approaches concentrating mainly on a pionless theory. Cutoff EFT for the exactly solvable toy model is discussed in section III. Finally, the findings of our work are briefly summarized in section IV.

\section{KSW VERSUS WEINBERG'S APPROACH}

For very low energies the effective non-relativistic Lagrangian relevant for S-wave nucleonnucleon scattering can be written as [1, 2, 7]:

$$
\mathcal{L}=N^{\dagger}\left[i \partial_{t}+\frac{\nabla^{2}}{2 m}\right] N-\frac{C_{S}}{2}\left(N^{\dagger} N\right)^{2}-\frac{C_{T}}{2}\left(N^{\dagger} \boldsymbol{\sigma} N\right)^{2}-\frac{C_{2}}{2}\left(N^{\dagger} \nabla^{2} N\right)\left(N^{\dagger} N\right)+\text { h.c. }+\ldots,
$$

where the nucleonic field $N$ is a two-component spinor in spin and isotopic spin spaces and $\boldsymbol{\sigma}$ are the Pauli matrices acting on spin indices. Further, $m$ is the nucleon mass and $C_{T}, C_{S}$ 
and $C_{2}$ are low energy coupling constants. The LO contribution to the NN potential in the ${ }^{1} S_{0}$ partial wave is

$$
V_{0}\left(p, p^{\prime}\right)=C_{S}-3 C_{T}=C,
$$

while the NLO one has the form:

$$
V_{2}\left(p, p^{\prime}\right)=C_{2}\left(p^{2}+p^{\prime 2}\right)
$$

In Weinberg's approach, the scattering amplitude is obtained by solving the LippmannSchwinger equation. For the potential $V_{0}+V_{2}$, the well-known solution for the on-theenergy-shell $T$-matrix reads, see e. g. [46],

$$
T=\frac{C+C_{2}^{2} I_{5}^{\Lambda}+k^{2} C_{2}\left(2-C_{2} I_{3}^{\Lambda}\right)}{\left(1-C_{2} I_{3}^{\Lambda}\right)^{2}-\left[C+C_{2}^{2} I_{5}^{\Lambda}+k^{2} C_{2}\left(2-C_{2} I_{3}^{\Lambda}\right)\right] I^{\Lambda}(k)},
$$

with the cutoff-regularized loop integrals defined as

$$
\begin{aligned}
I_{n}^{\Lambda} & =-\frac{m}{(2 \pi)^{3}} \int d^{3} l l^{n-3} \theta(\Lambda-l)=-\frac{m \Lambda^{n}}{2 n \pi^{2}}, \text { with } n=1,3,5, \\
I^{\Lambda}(k) & =\frac{m}{(2 \pi)^{3}} \int \frac{d^{3} l \theta(\Lambda-l)}{k^{2}-l^{2}+i \eta}=I_{1}^{\Lambda}-\frac{i m k}{4 \pi}-\frac{m k}{4 \pi^{2}} \ln \frac{\Lambda-k}{\Lambda+k},
\end{aligned}
$$

where $k$ refers to the on-shell momentum in the NN center-of-mass system and the last equation is valid for $k<\Lambda$. To renormalize (2.4) we divide loop integrals into the divergent and finite parts and take the limit $\Lambda \rightarrow \infty$ :

$$
\begin{aligned}
I_{n} & \equiv \lim _{\Lambda \rightarrow \infty} I_{n}^{\Lambda}=\lim _{\Lambda \rightarrow \infty}\left(I_{n}^{\Lambda}+\frac{m \mu_{n}^{n}}{2 n \pi^{2}}\right)-\frac{m \mu_{n}^{n}}{2 n \pi^{2}} \equiv \Delta_{n}\left(\mu_{n}\right)+I_{n}^{R}\left(\mu_{n}\right), \text { with } n=3,5, \\
I(k) & \equiv \lim _{\Lambda \rightarrow \infty} I^{\Lambda}(k)=\lim _{\Lambda \rightarrow \infty}\left(I_{1}^{\Lambda}+\frac{m \mu}{2 \pi^{2}}\right)+\left[-\frac{m \mu}{2 \pi^{2}}-\frac{i m k}{4 \pi}\right] \equiv \Delta(\mu)+I^{R}(\mu, k) .
\end{aligned}
$$

Here, $\Delta_{n}\left(\mu_{n}\right)$ and $\Delta(\mu)$ denote the divergent parts of the loop integrals while $I_{n}^{R}\left(\mu_{n}\right)$ and $I^{R}(\mu, k)$ are finite. The splitting of loop integrals in Eq. (2.6) is not unique. The freedom in the choice of renormalization conditions is parameterized by $\mu$ and $\mu_{n}$. The divergent parts $\Delta_{n}\left(\mu_{n}\right)$ and $\Delta(\mu)$ are to be canceled by contributions of counterterms. To absorb all appearances of $\Delta_{n}\left(\mu_{n}\right)$ and $\Delta(\mu)$ in Eq. (2.4) one needs to include contributions of an infinite number of counterterms of increasingly higher orders in powers of momenta [24]. While it is impossible to write down these counterterms explicitly, one can take their contributions into account by dropping $\Delta_{n}\left(\mu_{n}\right)$ and $\Delta(\mu)$ terms and replacing $C$ and $C_{2}$ by renormalized couplings. The subtracted (renormalized) amplitude reads:

$$
T=\frac{C_{R}+\left(C_{2}^{R}\right)^{2} I_{5}^{R}\left(\mu_{5}\right)+k^{2} C_{2}^{R}\left(2-C_{2}^{R} I_{3}^{R}\left(\mu_{3}\right)\right)}{\left(1-C_{2}^{R} I_{3}^{R}\left(\mu_{3}\right)\right)^{2}-\left[C_{R}+\left(C_{2}^{R}\right)^{2} I_{5}^{R}\left(\mu_{5}\right)+k^{2} C_{2}^{R}\left(2-C_{2}^{R} I_{3}^{R}\left(\mu_{3}\right)\right)\right] I^{R}(\mu, k)} .
$$

Note that we are free to fix the freedom in finite parts of loop integrals. Any scheme that puts an effective cut-off of the order of external momenta is equally good from the EFT point of view. We also emphasize that Eq. (2.7) is not obtained from (2.4) by just expressing $C$ and $C_{2}$ in terms of renormalized coupling constants since these two low-energy constants are insufficient to absorb all occuring divergences. The renormalized couplings $C_{R}$ and 
$C_{2}^{R}$ depend on the renormalization conditions through the renormalization group, but this implicit dependence does not cancel completely the explicit dependence of the amplitude on $\mu$ and $\mu_{n}$. However, different choices are equivalent up to the order of accuracy of the calculation, provided that the chosen renormalization conditions respect power counting.

We now turn to the KSW approach [9], where the T-matrix up to subleading order is given by ${ }^{1}$ :

$$
T=\frac{C}{1-C I^{\Lambda}(k)}+\frac{2 k^{2} C_{2}+2 C C_{2} I_{3}^{\Lambda}}{\left[1-C I^{\Lambda}(k)\right]^{2}} .
$$

One can absorb all divergences appearing in the above expression by expressing the bare couplings $C$ and $C_{2}$ in terms of renormalized ones $C_{R}\left(=C_{R}\left(\mu, \mu_{3}\right)\right)$ and $C_{2}^{R}\left(=C_{2}^{R}\left(\mu, \mu_{3}\right)\right)$. The complete functional dependencies $C \equiv C\left(C_{R}, C_{2}^{R}\right)$ and $C_{2} \equiv C_{2}\left(C_{R}, C_{2}^{R}\right)$ can be found in Ref. [25]. For our purposes, it is sufficient to expand these expressions in powers of $C_{2}^{R}$ which leads to

$$
\begin{aligned}
C & =\frac{C_{R}}{1+C_{R} \Delta(\mu)}+\frac{2 C_{R} C_{2}^{R} I_{3}^{R}\left(\mu_{3}\right)}{\left[1+C_{R} \Delta(\mu)\right]^{2}}-\frac{2 C_{R} C_{2}^{R} I_{3}}{\left[1+C_{R} \Delta(\mu)\right]^{3}}+\cdots, \\
C_{2} & =\frac{C_{2}^{R}}{\left[1+C_{R} \Delta(\mu)\right]^{2}}+\cdots .
\end{aligned}
$$

In the KSW approach, the first term in Eq. (2.9) is treated non-perturbatively while all other terms in Eqs. (2.9) and (2.10) are taken into account perturbatively, order-by-order. Substituting Eqs. (2.9) and (2.10) into (2.8) we obtain a finite renormalized expression:

$$
T=\frac{C_{R}}{1-C_{R} I^{R}(\mu, k)}+\frac{2 C_{R} C_{2}^{R} I_{3}^{R}\left(\mu_{3}\right)}{\left[1-C_{R} I^{R}(\mu, k)\right]^{2}}+\frac{2 k^{2} C_{2}^{R}}{\left[1-C_{R} I^{R}(\mu, k)\right]^{2}}+\cdots .
$$

If we choose $\Delta_{3} \equiv I_{3}$ (i.e. we set $\mu_{3}=0$ ), the explicit dependence on $\mu$ can be completely compensated by implicit dependence of running couplings $C_{R}$ and $C_{2}^{R}$ at any fixed order in the EFT expansion. This is analogous to Refs. [9, 10] where the dimensional regularization in combination with power divergence subtraction (PDS) scheme is used. Complete orderby-order renormalization scale-independence cannot be achieved for any other choice of $\mu_{3}$. Indeed, if the amplitude were $\mu$ - and $\mu_{3}$-independent order-by-order, the third term in Eq. (2.11) should satisfy this condition by itself. Denoting this term with $t_{3}$ we obtain

$$
\sqrt{\frac{2 k^{2}}{t_{3}}}=\left[\frac{1}{C_{R}}-I^{R}(\mu, k)\right] \frac{C_{R}}{\sqrt{C_{2}^{R}}} .
$$

As the integral $I^{R}(\mu, k)$ has an imaginary part which is renormalization scale independent, it follows from Eq. (2.12) that $C_{R} / \sqrt{C_{2}^{R}}$ must be renormalization scale independent. If this were the case, both $C_{R}$ and $C_{2}^{R}$ must be $\mu_{3}$-independent as it is easily seen from the real part of the same equation. However, in this case the explicit $\mu_{3}$-dependence of the second term in Eq. (2.11) cannot be canceled by running of the coupling constants $C_{R}$ and $C_{2}^{R}$. We are forced to conclude that the amplitude cannot be renormalization scale-independent order-by-order.

\footnotetext{
${ }^{1}$ It results from Eq. (2.4) by expanding in powers of $C_{2}$ and keeping the first two terms.
} 
It is instructive to verify that the amplitude is indeed renormalization-scale independent up to terms of order $\mathcal{O}\left(\left(C_{2}^{R}\right)^{2}\right)$. By differentiating the expressions of the bare couplings in terms of renormalized ones, $C=C\left(C_{R}, C_{2}^{R}\right)$ and $C_{2}=C\left(C_{R}, C_{2}^{R}\right)$ [25], with respect to renormalization scales one obtains the corresponding renormalization group equations for renormalized couplings. For the beta-functions to first order in $C_{2}^{R}$ these equations read:

$$
\begin{aligned}
\frac{\partial C_{R}}{\partial \mu} & =\frac{m}{2 \pi^{2}} C_{R}^{2}+\frac{m^{2} \mu_{3}^{3}}{6 \pi^{4}} C_{R}^{2} C_{2}^{R}, \\
\frac{\partial C_{R}}{\partial \mu_{3}} & =\frac{m \mu_{3}^{2}}{\pi^{2}} C_{R} C_{2}^{R}, \\
\frac{\partial C_{2}^{R}}{\partial \mu} & =\frac{m}{\pi^{2}} C_{R} C_{2}^{R}, \\
\frac{\partial C_{2}^{R}}{\partial \mu_{3}} & =0 .
\end{aligned}
$$

We were unable to solve Eqs. (2.13) in a closed form but obtained the expantion of the solution in powers of $C_{2}^{R \text { : }}$

$$
\begin{aligned}
C_{R}\left(\mu, \mu_{3}\right) & =\frac{2 \pi^{2} C_{R}(0,0)}{2 \pi^{2}-\mu m C_{R}(0,0)}+\frac{8 \pi^{4} \mu_{3}^{3} m C_{R}(0,0) C_{2}^{R}(0)}{3\left(2 \pi^{2}-\mu m C_{R}(0,0)\right)^{3}}+\cdots \\
C_{2}^{R}(\mu) & =\frac{4 \pi^{4} C_{2}^{R}(0)}{\left(2 \pi^{2}-\mu m C_{R}(0,0)\right)^{2}}+\cdots
\end{aligned}
$$

Substituting Eq. (2.14) into Eq. (2.11) leads to

$$
\begin{aligned}
T & =-\frac{2 \pi^{2} C_{R}(0,0)}{2 \pi^{2}-C_{R}(0,0)\left[m \mu+2 \pi^{2} I^{R}(\mu, k)\right]}+\frac{8 C_{2}^{R}(0) \pi^{4} k^{2}}{\left\{2 \pi^{2}-C_{R}(0,0)\left[m \mu+2 \pi^{2} I^{R}(\mu, k)\right]\right\}^{2}} \\
& +\cdots
\end{aligned}
$$

which is renormalization scheme independent up to the considered order.

In case of the KSW approach, even using the PDS scheme, the residual renormalization scale dependence is present in running coupling constants if pions are included as explicit degrees of freedom. The LO renormalized (running) coupling constant in the ${ }^{1} S_{0}$ partial wave given in Ref. [10] reads

$$
C_{0}^{\left(1 S_{0}\right)}(\mu)=-\frac{4 \pi}{\mu m}\left(\frac{1}{1-\left[\mu\left(a+1 / \Lambda_{N N}\right)\right]^{-1}}+\frac{\mu}{\Lambda_{N N}}\right), \quad \Lambda_{N N}=\frac{8 \pi f^{2}}{g_{A}^{2} m},
$$

where $a$ is the ${ }^{1} S_{0}$ scattering length, $f$ denotes the pion decay constant normalized to be $f=132 \mathrm{MeV}$ and $g_{A}$ is the axial-vector pion-nucleon coupling constant. In the expansion of $C_{0}^{\left({ }^{1} S_{0}\right)}(\mu)$ in powers of $g_{A}$, the renormalization scale dependence is present to all orders. This dependence is canceled by an infinite number of higher-order terms in the expansion of the amplitude. Notice that in KSW approach the appearance of the residual renormalization scale dependence has a different origin compared to the Weinberg approach. There it arises from the explicit renormalization scheme dependence of loop integrals due to missing contributions of the corresponding higher-order renormalized coupling constants.

To summarize the above considerations, renormalization scheme dependence is present explicitly in loop contributions and implicitly in running coupling constants. These two types 
of dependence exactly cancel each other in the full amplitude. Due to the order-by-order calculations in the KSW approach, there is a residual renormalization scheme dependence generated by running couplings. On the other hand, in the case of Weinberg's approach, the residual renormalization scale dependence arises from loop contributions. There is no conceptual difference between the two approaches from the point of view of the renormalization scale dependence. If one approach is inconsistent, then so is the other. In fact both are conceptually as consistent as perturbative QCD is. Of course, the crucial issue is to choose an optimal renormalization scheme (if such a scheme exists at all). In perturbative QCD, when expressed in terms of the running coupling, the full amplitudes are independent of the renormalization scale $\mu$. Perturbative expressions are, however, $\mu$-independent only up to the order of accuracy of the calculation. The residual $\mu$-dependence is canceled by higher-order terms in perturbation theory. At high energies one could choose renormalization scale much smaller than the characteristic scale in a process under consideration. This would generate a large value of the running coupling constant and, at the same time, lead to large coefficients in the perturbative series. The failure of this kind of perturbative scheme does not mean the failure of perturbation theory in high-energy QCD in general. The only problem is that for such an inappropriate choice of the renormalization scheme, "higher-order" $\mu$-dependent terms play a crucial role and are by no means suppressed. EFT for few nucleons is conceptually similar. Although observables are calculated by solving the corresponding dynamical equations, one is still doing perturbative calculations with respect to the chiral expansion. While the full amplitude is renormalization scheme independent, truncated expressions at any finite order are generally not.

We now switch to our next topic and consider Weinberg's approach based on a finite cutoff rather than subtractive renormalization. In particular, we are interested in the implications of taking the cutoff value very large. To keep trace of the loop expansion, we first rewrite Eq. (2.4) by showing explicitly factors of $\hbar$ :

$$
T=\frac{C+C_{2}^{2} \hbar I_{5}+k^{2} C_{2}\left(2-C_{2} \hbar I_{3}\right)}{\left(1-C_{2} \hbar I_{3}\right)^{2}-\left[C+C_{2}^{2} \hbar I_{5}+k^{2} C_{2}\left(2-C_{2} \hbar I_{3}\right)\right] \hbar I(k)} .
$$

The bare coupling constants $C$ and $C_{2}$ can be expressed in terms of the scattering length $a$ and the effective range $r$ by matching the amplitude in Eq. (2.17) to the first two terms in the effective range expansion

$$
\Re\left(T^{-1}\right)=-\frac{m}{4 \pi}\left(-\frac{1}{a}+\frac{1}{2} r k^{2}+\ldots\right),
$$

which leads to the following expressions

$$
\begin{gathered}
C=C(a, r, \Lambda)=\frac{6 \pi^{2}\left[a^{2} \hbar \Lambda^{3} m(64 \hbar-3 \pi \Lambda r)-6\left(D-3 \pi^{2} \Lambda m\right)-62 \pi a \hbar \Lambda^{2} m\right]}{5 \hbar \Lambda^{2} m^{2}\left[a^{2} \hbar \Lambda^{2}(16 \hbar-\pi \Lambda r)-12 \pi a \hbar \Lambda+3 \pi^{2}\right]}, \\
C_{2}=C_{2}(a, r, \Lambda)=-\frac{6 \pi^{2}\left[-D+a^{2} \hbar m \Lambda^{3}(16 \hbar-\pi r \Lambda)-12 \pi a \hbar m \Lambda^{2}+3 \pi^{2} m \Lambda\right]}{\hbar m^{2} \Lambda^{4}\left[a^{2} \hbar \Lambda^{2}(16 \hbar-\pi r \Lambda)-12 \pi a \hbar \Lambda+3 \pi^{2}\right]}
\end{gathered}
$$

with $D$ defined as

$$
D=\sqrt{3} \sqrt{\Lambda^{2} m^{2}(\pi-2 a \hbar \Lambda)^{2}\left(a^{2} \hbar \Lambda^{2}(16 \hbar-\pi \Lambda r)-12 \pi a \hbar \Lambda+3 \pi^{2}\right)} .
$$


Substituting the resulting expressions for the bare couplings $C(a, r, \Lambda)$ and $C_{2}(a, r, \Lambda)$ back into Eq. (2.17), we obtain for the inverse scattering amplitude

$$
\begin{aligned}
T^{-1}= & \frac{m}{4 \pi^{2} a\left[a\left(\pi k^{2} r \Lambda-4 \hbar\left(k^{2}+\Lambda^{2}\right)\right)+2 \pi \Lambda\right]}\left\{2 \Lambda\left[a^{2} \hbar k^{2}(\pi r \Lambda-4 \hbar)-2 \pi a \hbar \Lambda+\pi^{2}\right]\right. \\
& \left.+a \hbar k \ln \frac{\Lambda-k}{\Lambda+k}\left[a\left(\pi k^{2} r \Lambda-4 \hbar\left(k^{2}+\Lambda^{2}\right)\right)+2 \pi \Lambda\right]\right\}+i \hbar \frac{m k}{4 \pi} .
\end{aligned}
$$

Although this expression for $T^{-1}$ possesses a finite limit as $\Lambda \rightarrow \infty$ which, as desired, correctly reproduces the first two terms in the effective range expansion (ERE),

$$
T^{-1}=-\frac{m}{4 \pi}\left(-\frac{1}{a}+\frac{1}{2} r k^{2}-i \hbar k\right)+\mathcal{O}\left(\Lambda^{-1}\right) .
$$

taking this limit without including all relevant contributions of counterterms is a meaningless procedure within an EFT [24]. Not surprisingly, one encounters pathologies, such as e. g. the coupling $C_{2}$ becoming complex for positive values of the effective range, see also Ref. [47].

To further explore the large-cutoff limit, we expand the obtained expressions for the bare LECs in Eq. (2.19) in powers of $\hbar$ which leads to

$$
\begin{aligned}
C & =\frac{4 \pi a}{m}+\hbar \frac{3 a^{4} r^{2} \Lambda^{5}+40 a^{3} r \Lambda^{3}+240 a^{2} \Lambda}{30 m}+\mathcal{O}\left(\hbar^{2}\right), \\
C_{2} & =\frac{\pi a^{2} r}{m}+\hbar \frac{a^{4} r^{2} \Lambda^{4}+16 a^{3} r \Lambda^{2}-16 a^{2}}{4 m \Lambda}+\mathcal{O}\left(\hbar^{2}\right) .
\end{aligned}
$$

Making use of the standard splitting of bare quantities into renormalized ones and counterterms,

$$
\begin{gathered}
C=C^{R}+\sum_{k=1}^{\infty} \hbar^{k} \delta C_{k}, \\
C_{2}=C_{2}^{R}+\sum_{k=1}^{\infty} \hbar^{k} \delta C_{2 k}
\end{gathered}
$$

we identify the renormalized low-energy constants in this particular scheme with

$$
C_{R}=\frac{4 \pi a}{m}, \quad C_{2}^{R}=\frac{\pi a^{2} r}{m} .
$$

Inverting the above expressions and replacing in Eq. (2.21) the scattering length and the effective range by $C_{R}$ and $C_{2}^{R}$, the inverse amplitude can be re-written in terms of renormalized coupling constants. To see what does the $\Lambda \rightarrow \infty$ limit correspond to in the language of the EFT diagrams, we regard the loop expansion of the amplitude (thus we reproduce the perturbative series summed up by iterating the LS equation):

$$
\begin{aligned}
T & =C_{R}+2 C_{2}^{R} k^{2}-i \hbar \frac{m k}{4 \pi}\left(C_{R}+2 C_{2}^{R} k^{2}\right)^{2} \\
& +\hbar \frac{2 m k^{4}}{\pi^{2}}\left[-\left(C_{2}^{R}\right)^{2} \Lambda+\left(C_{2}^{R}\right)^{2} k^{2} \Lambda^{-1}+C_{2}^{R} C_{R} \Lambda^{-1}+\mathcal{O}\left(\Lambda^{-2}\right)\right]+\cdots
\end{aligned}
$$


where ellipsis refer to higher-order terms in the loop expansion. For momenta $k \gtrsim 1 / a$ we cannot truncate the loop expansion in Eq. (2.26) at any finite order (i.e. in the language of Feynman diagrams we need to sum up an infinite number of them). For our demonstrating purposes we consider here the case when $r \ll a$. With renormalized couplings of Eq. (2.25) the small parameter of the EFT expansion is given by $k^{2} / m_{s}^{2} \sim 2 C_{2}^{R} k^{2} / C_{R}=a r k^{2} / 2$, i.e. the hard scale of the problem is $m_{s} \sim 1 / \sqrt{r a}{ }^{2}$ The term linear in $\Lambda$ in the second line of Eq. (2.26) violates the dimensional power counting and, for very large values of $\Lambda$ (much larger than $m_{s}$ ), yields the numerically dominant contribution at one loop order, instead of being absorbed into redefinition of higher-order coupling constants or, equivalently, being subtracted. The situation is similar at higher orders in the loop expansion. Hence, one completely looses the power counting which the EFT is based on: terms which are supposed to be subtracted yield dominant contributions to the amplitude. On the other hand, if $\Lambda$ is taken of the order of the hard scale in the problem, i.e. $\Lambda \sim m_{s}$, the term linear in $\Lambda$ in the second line of Eq. (2.26) appears to be of order three and is beyond the accuracy of the considered calculation. Notice that reducing the value of $\Lambda$ considerably below the hard scale would lead to large cutoff artefacts generated by terms with negative powers of $\Lambda$. One is, therefore, forced to conclude that in the cutoff theory, $\Lambda$ should ideally be chosen of the order of the hard scale in the problem.

\section{CUTOFF EFT: RENORMALIZATION VERSUS "PERATIZATION"}

In this section we further explore and extend the above ideas by considering effective theory for an exactly solvable quantum mechanical model for two nucleons interacting via the long- and short-range forces. This may be regarded as a toy model for chiral EFT in the twonucleon sector. We employ both the subtractive renormalization and the cutoff formulation of the resulting effective theory and discuss the similarities and differences between these two approaches. We also explore the consequences of taking very large values of the cutoff in this model.

\section{A. The model}

We consider two nucleons in the spin-singlet S-wave interacting via the two-range separable potential

$$
V\left(p, p^{\prime}\right)=v_{l} F_{l}(p) F_{l}\left(p^{\prime}\right)+v_{s} F_{s}(p) F_{s}\left(p^{\prime}\right), \quad F_{l}(p) \equiv \frac{\sqrt{p^{2}+m_{s}^{2}}}{p^{2}+m_{l}^{2}}, \quad F_{s}(p) \equiv \frac{1}{\sqrt{p^{2}+m_{s}^{2}}},
$$

where the subscripts $l$ and $s$ refer to long- and short-range interactions and the mass scales $m_{l}$ and $m_{s}$ fulfill the condition $m_{l} \ll m_{s}$. Further, the dimensionless quantities $v_{l}$ and $v_{s}$ denote the strengths of the long- and short-range interactions, respectively. Our choice of the explicit form of $F_{l, s}(p)$ is entirely motivated by the simplicity of calculations. The reader may verify that all conclusions reached in this section remain valid if one chooses, for example, $F_{l, s}(p) \propto 1 /\left(p^{2}+m_{l, s}^{2}\right)$. In this case, however, one will need to go to subleading

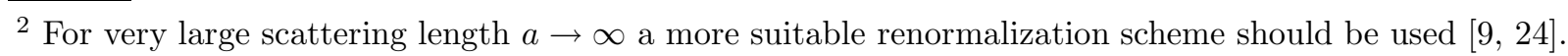


order in the EFT expansion in order to explore the "peratization" procedure which would make the calculations considerably more involved.

For an interaction of a separable type, the off-shell T-matrix can be easily calculated analytically by solving the corresponding Lippmann-Schwinger equation

$$
T\left(p, p^{\prime} ; k\right)=V\left(p, p^{\prime}\right)+4 \pi \int \frac{l^{2} d l}{(2 \pi)^{3}} V(p, l) \frac{m}{k^{2}-l^{2}+i \epsilon} T\left(l, p^{\prime} ; k\right),
$$

where $m$ is the nucleon mass and $k$ corresponds to the on-shell momentum which is related to the two-nucleon center-of-mass energy via $E_{\mathrm{CMS}}=k^{2} / m$. The phase shift $\delta(k)$ can be obtained from the on-the-energy-shell T-matrix elements via

$$
T(k, k ; k)=-\frac{4 \pi}{m} \frac{1}{k \cot \delta(k)-i k} .
$$

Here and in the following, we are particularly interested in the coefficients entering the effective range expansion

$$
k \cot \delta(k)=-\frac{1}{a}+\frac{1}{2} r k^{2}+v_{2} k^{4}+v_{3} k^{6}+\ldots,
$$

with $a, r$ and $v_{i}$ referring to the scattering length, effective range and the so-called shape parameters. The coefficients in the ERE generally scale with the mass corresponding to the long-range interaction which gives rise to the first left-hand cut in the T-matrix. Notice that the scattering length can be tuned to any value by adjusting the strength of the interaction. The coefficients in the ERE can be expanded in powers of $m_{l} / m_{s}$ leading to the "chiral" expansion:

$$
\begin{aligned}
a & =\frac{1}{m_{l}}\left(\alpha_{a}^{(0)}+\alpha_{a}^{(1)} \frac{m_{l}}{m_{s}}+\alpha_{a}^{(2)} \frac{m_{l}^{2}}{m_{s}^{2}}+\ldots\right), \\
r & =\frac{1}{m_{l}}\left(\alpha_{r}^{(0)}+\alpha_{r}^{(1)} \frac{m_{l}}{m_{s}}+\alpha_{r}^{(2)} \frac{m_{l}^{2}}{m_{s}^{2}}+\ldots\right), \\
v_{i} & =\frac{1}{m_{l}^{2 i-1}}\left(\alpha_{v_{i}}^{(0)}+\alpha_{v_{i}}^{(1)} \frac{m_{l}}{m_{s}}+\alpha_{v_{i}}^{(2)} \frac{m_{l}^{2}}{m_{s}^{2}}+\ldots\right),
\end{aligned}
$$

where $\alpha_{a}^{(m)}, \alpha_{r}^{(m)}$ and $\alpha_{v_{i}}^{(m)}$ are dimensionless constants whose values are determined by the specific form of the interaction potential. We fine tune the strengths of the long- and shortrange interactions in such a way that they generate scattering lengths of a natural size. More precisely, we require that the scattering length takes the value $a=\alpha_{l} / m_{l}\left(a=\alpha_{s} / m_{s}\right)$ with a dimensionless constant $\left|\alpha_{l}\right| \sim 1\left(\left|\alpha_{s}\right| \sim 1\right)$ when the short-range (long-range) interaction is switched off. This leads to

$$
v_{l}=-\frac{8 \pi m_{l}^{3} \alpha_{l}}{m\left(\alpha_{l} m_{s}^{2}+m_{l}^{2} \alpha_{l}-2 m_{s}^{2}\right)}, \quad v_{s}=-\frac{4 \pi m_{s} \alpha_{s}}{m\left(\alpha_{s}-1\right)} .
$$

One then finds the following expressions for the first three terms in the "chiral" expansion in Eq. (3.5).

- Scattering length:

$$
\alpha_{a}^{(0)}=\alpha_{l}, \quad \alpha_{a}^{(1)}=\left(\alpha_{l}-1\right)^{2} \alpha_{s}, \quad \alpha_{a}^{(2)}=\left(\alpha_{l}-1\right)^{2} \alpha_{l} \alpha_{s}^{2} .
$$


- Effective range:

$$
\begin{aligned}
& \alpha_{r}^{(0)}=\frac{3 \alpha_{l}-4}{\alpha_{l}}, \quad \alpha_{r}^{(1)}=\frac{2\left(\alpha_{l}-1\right)\left(3 \alpha_{l}-4\right) \alpha_{s}}{\alpha_{l}^{2}}, \\
& \alpha_{r}^{(2)}=\frac{\left(\alpha_{l}-1\right)\left(3 \alpha_{l}-4\right)\left(5 \alpha_{l}-3\right) \alpha_{s}^{2}+\left(2-\alpha_{l}\right) \alpha_{l}^{2}}{\alpha_{l}^{3}} .
\end{aligned}
$$

- First shape parameter:

$$
\begin{aligned}
& \alpha_{v_{2}}^{(0)}=\frac{\alpha_{l}-2}{2 \alpha_{l}}, \quad \alpha_{v_{2}}^{(1)}=\frac{\left[\alpha_{l}\left(13 \alpha_{l}-36\right)+24\right] \alpha_{s}}{4 \alpha_{l}^{2}}, \\
& \alpha_{v_{2}}^{(2)}=\frac{\left\{\alpha_{l}\left[\alpha_{l}\left(46 \alpha_{l}-159\right)+174\right]-60\right\} \alpha_{s}^{2}-4\left(\alpha_{l}-2\right) \alpha_{l}^{2}}{4 \alpha_{l}^{3}} .
\end{aligned}
$$

- Second shape parameter:

$$
\begin{aligned}
& \alpha_{v_{3}}^{(0)}=0, \quad \alpha_{v_{3}}^{(1)}=\frac{\left(\alpha_{l}-2\right)\left(3 \alpha_{l}-4\right) \alpha_{s}}{2 \alpha_{l}^{2}}, \\
& \alpha_{v_{3}}^{(2)}=\frac{\left(3 \alpha_{l}-4\right)\left[\alpha_{l}\left(25 \alpha_{l}-68\right)+40\right] \alpha_{s}^{2}-4\left(\alpha_{l}-2\right) \alpha_{l}^{2}}{8 \alpha_{l}^{3}} .
\end{aligned}
$$

Notice that the ERE is not applicable in the case $\alpha_{l} \rightarrow 0$ as follows immediately from the considerations based on the Born approximation. We further stress that in our model the leading terms in the $m_{l} / m_{s}$-expansion of the ERE coefficients are completely fixed by the long-range interaction. The scenario realized corresponds to a strong (at momenta $k \sim m_{l}$ ) long-range interaction which needs to be treated non-perturbatively and a weak short-range interaction which can be taken into account perturbatively. We, however, emphasize that this particular hierarchy is not important for our purposes.

\section{B. KSW-like approach and the low-energy theorems}

Various coefficients in the ERE are correlated with each other as a consequence of the long-range interaction. In the context of effective (field) theory, such correlations are to be regarded as low-energy theorems. They have been discussed for the realistic case of nucleonnucleon interaction within the KSW scheme in Refs. [16, 17, 18] and were shown to fail badly in the ${ }^{1} S_{0}$ and ${ }^{3} S_{1}-{ }^{3} D_{1}$ channels. This failure is a clear signal towards the non-perturbative nature of the one-pion exchange in these channels. At the qualitative level, the low-energy theorems in the pionful EFT simply reflect the hierarchy $M_{\pi} \ll \Lambda_{\text {hard }}$ between the soft and hard scales in the problem, which set the upper bounds for the convergence radii of the ERE and chiral expansion, respectively. We will specify the precise meaning of the low-energy theorems for the case at hand in the following.

We now develop EFT for the model specified above by keeping the long-range interaction and replacing the short-range potential by a series of contact zero-range interactions:

$$
V_{\text {short }}\left(p, p^{\prime}\right)=C_{0}+C_{2}\left(p^{2}+p^{\prime 2}\right)+\ldots,
$$

where $C_{2 n}$ are low-energy constants. We begin with the most convenient and elegant formulation which respects the standard dimensional power counting. To achieve that we use 

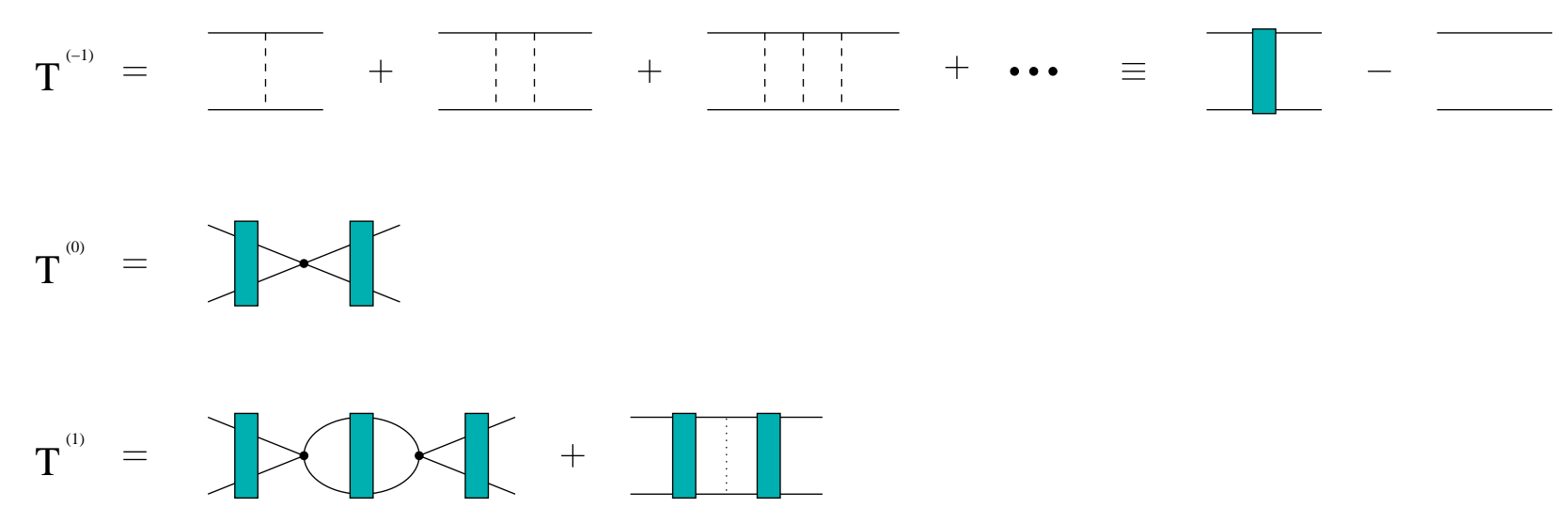

FIG. 1: Leading, next-to-leading and next-to-next-to-leading order contributions to the scattering amplitude in the KSW-like approach. The solid lines denote nucleons while the dashed ones represent an insertion of the lowest-order long-range interaction. Solid dots (dotted lines) denote an insertion of the lowest-order contact interaction $\propto C_{0}$ (subleading contribution to the long-range interaction).

subtractive renormalization for all divergent integrals and choose the subtraction constant $\mu \sim m_{l}$. We expand the long-range interaction in powers of $p / m_{s}$ in order to prevent the appearance of positive powers of the large scale in the expressions for renormalized loop diagrams which would spoil the power counting. We also expand the strength of the long-range interaction $v_{l}$ in Eq. (3.6) in powers of $m_{l} / m_{s}$ although this is not necessary to maintain the power counting. Here and in the following, we refer to this approach as KSW-like. To be specific, we compute the first few terms in the $Q / \lambda$-expansion of the T-matrix with $Q=\left\{k, m_{l}, \mu\right\}$ and $\lambda=\left\{m_{s}, m\right\}$. Notice that the natural size of the short-range effects in our model suggests the scaling of the short-range interactions in agreement with the naive dimensional analysis, i.e. $C_{2 n} \sim Q^{0}$. The leading contribution to the T-matrix at order $Q^{-1}$ is generated by the leading term in the $Q / \lambda$-expansion of the long-range interaction

$$
\begin{aligned}
V_{\text {long }}\left(p, p^{\prime}\right) & =v_{l} F_{l}(p) F_{l}\left(p^{\prime}\right) \\
& \simeq-\frac{8 \pi m_{l}^{3} \alpha_{l}}{m\left(\alpha_{l}-2\right)\left(p^{2}+m_{l}^{2}\right)\left(p^{\prime 2}+m_{l}^{2}\right)}\left[1-\frac{\alpha_{l} m_{l}^{2}}{\left(\alpha_{l}-2\right) m_{s}^{2}}+\frac{p^{2}}{2 m_{s}^{2}}+\frac{p^{\prime 2}}{2 m_{s}^{2}}+\mathcal{O}\left(\frac{Q^{4}}{\lambda^{4}}\right)\right]
\end{aligned}
$$

which scales as $Q^{-1}$ and, therefore, needs to be summed up to an infinite order, see Fig. 1). This leads to the following expression for the on-the-energy shell T-matrix:

$$
T^{(-1)}=-\frac{8 \pi m_{l}^{3} \alpha_{l}}{m\left(k-i m_{l}\right)^{2}\left[k^{2}\left(\alpha_{l}-2\right)+2 i k m_{l}\left(\alpha_{l}-2\right)+2 m_{l}^{2}\right]}
$$

from which one deduces

$$
\begin{aligned}
k \cot \delta & =-\frac{4 \pi}{m} \frac{1}{T^{(-1)}}+i k \\
& =-\frac{m_{l}}{\alpha_{l}}+\frac{\left(3 \alpha_{l}-4\right)}{2 m_{l} \alpha_{l}} k^{2}+\frac{\left(\alpha_{l}-2\right)}{2 m_{l}^{3} \alpha_{l}} k^{4} .
\end{aligned}
$$


Not surprisingly, one observes that the leading terms in the expansion of the ERE coefficients in Eq. (3.5) are correctly reproduced. The first correction to the scattering amplitude at order $Q^{0}$ is given by the leading-order contact interaction dressed with the iterated leading long-range interaction as visualized in Fig. 1, One finds

$$
T^{(0)}=\frac{C_{0}\left(k+i m_{l}\right)^{2}\left[k^{2}\left(\alpha_{l}-2\right)+2 m_{l}^{2}\left(\alpha_{l}-1\right)\right]^{2}}{\left(k-i m_{l}\right)^{2}\left[k^{2}\left(\alpha_{l}-2\right)+2 i k m_{l}\left(\alpha_{l}-2\right)+2 m_{l}^{2}\right]^{2}} .
$$

Notice that all integrals entering $T^{(-1)}$ and $T^{(0)}$ are finite. The effective range function $k \cot \delta$ at $\mathrm{NLO}$ can be computed via

$$
k \cot \delta=-\frac{4 \pi}{m} \frac{1}{T^{(-1)}}\left(1-\frac{T^{(0)}}{T^{(-1)}}\right)+i k .
$$

The "chiral" expansion of the coefficients in the ERE results from expanding the right-hand side in this equation in powers of $k^{2}$ and, subsequently, in powers of $m_{l}$. The LEC $C_{0}$ can be determined from matching to $\alpha_{a}^{(1)}$ in Eq. (3.7) which yields

$$
C_{0}=\frac{4 \pi \alpha_{s}}{m m_{s}}
$$

This leads to the following predictions for $r, v_{2}$ and $v_{3}$ :

$$
\begin{aligned}
r^{\mathrm{NLO}} & =\frac{1}{m_{l}}\left[\frac{3 \alpha_{l}-4}{\alpha_{l}}+\frac{2\left(\alpha_{l}-1\right)\left(3 \alpha_{l}-4\right) \alpha_{s}}{\alpha_{l}^{2} m_{s}} m_{l}\right] \\
v_{2}^{\mathrm{NLO}} & =\frac{1}{m_{l}^{3}}\left[\frac{\left(\alpha_{l}-2\right)}{2 \alpha_{l}}+\frac{\left(\alpha_{l}\left(13 \alpha_{l}-36\right)+24\right) \alpha_{s}}{4 \alpha_{l}^{2} m_{s}} m_{l}\right] \\
v_{3}^{\mathrm{NLO}} & =\frac{1}{m_{l}^{4}} \frac{\left(\alpha_{l}-2\right)\left(3 \alpha_{l}-4\right) \alpha_{s}}{2 \alpha_{l}^{2} m_{s}} .
\end{aligned}
$$

One observes that $\alpha_{r}^{(1)}, \alpha_{v_{2}}^{(1)}$ and $\alpha_{v_{3}}^{(1)}$ are correctly reproduced at NLO. Using dimensional analysis it is easy to verify that, in fact, $\alpha_{v_{i}}^{(1)}$ for all $i$ must be reproduced correctly at this order.

Finally, at next-to-next-to-leading order (NNLO) one has to take into account the leading corrections to the long-range potential in Eq. (3.12) and the contribution due to once iterated leading-order contact term. Clearly, these contributions have to be dressed by the iterated leading long-range interaction, see Fig. 1. The contribution $\propto C_{0}^{2}$ involves a linearly divergent integral which we regularize with a cutoff $\Lambda \gg m_{l}$ :

$$
I_{1}^{\mathrm{reg}} \equiv 4 \pi m \int_{0}^{\Lambda} \frac{l^{2} d l}{(2 \pi)^{3}} \frac{1}{k^{2}-l^{2}+i \epsilon}=-\frac{m \Lambda}{2 \pi^{2}}-i \frac{m k}{4 \pi}+\mathcal{O}\left(\Lambda^{-1}\right) .
$$

We carry out renormalization by subtracting the divergent part of the integral $-m /\left(2 \pi^{2}\right) \int_{\mu}^{\Lambda} d l$, taking the limit $\Lambda \rightarrow \infty$,

$$
I_{1}^{\mathrm{reg}} \rightarrow I_{1}^{\mathrm{subtr}}=-\frac{m \mu}{2 \pi^{2}}-i \frac{m k}{4 \pi}
$$

and replacing the bare $C_{0}$ by the renormalized one $C_{0}(\mu)$. As already pointed out before, we choose $\mu \sim m_{l}$ in order to be consistent with the standard power counting based on 
the dimensional analysis. Clearly, the above procedure is exactly equivalent to the power divergence subtraction prescription utilized in the KSW framework. A simple calculation yields the following result for the sub-subleading contribution to the amplitude:

$$
\begin{aligned}
T^{(1)}= & \frac{\left(k+i m_{l}\right)^{2}}{4 \pi^{2} m m_{s}^{2}\left(k-i m_{l}\right)^{2}\left[k \alpha_{l}\left(k+2 i m_{l}\right)-2\left(k+i m_{l}\right)^{2}\right]^{2}}\left[-32 \pi^{3} k^{2} m_{l}^{3}\left(\alpha_{l}-2\right) \alpha_{l}\right. \\
& +\left(C_{0}(\mu)\right)^{2} m^{2} m_{s}^{2}\left[k^{2}\left(\alpha_{l}-2\right)+2 m_{l}^{2}\left(\alpha_{l}-1\right)\right]^{2} \\
& \left.\times \frac{\alpha_{l}\left[k^{2}(-2 \mu-i \pi k)+2 k(\pi k-2 i \mu) m_{l}+2 \pi m_{l}^{3}\right]+2(2 \mu+i \pi k)\left(k+i m_{l}\right)^{2}}{k \alpha_{l}\left(k+2 i m_{l}\right)-2\left(k+i m_{l}\right)^{2}}\right] .
\end{aligned}
$$

The LEC $C_{0}(\mu)$ can be written in terms of the perturbative expansion as follows

$$
C_{0}(\mu)=C_{0}^{(0)}+C_{0}^{(1)}(\mu)+\ldots,
$$

where the superscript refers to the power of the soft scale $Q$. The first term does not depend on $\mu$ and equals $C_{0}$ in Eq. (3.17). The $\mu$-dependence of $C_{0}^{(1)}(\mu)$ can be determined by solving the renormalization group equation

$$
\frac{d}{d \mu}\left[T^{(-1)}+T^{(0)}+T^{(1)}\right]=0
$$

One also needs one additional input parameter, such as e. g. $\alpha_{a}^{(2)}$, in order to fix the integration constant. This leads to

$$
C_{0}^{(1)}=\frac{8 \mu \alpha_{s}^{2}}{m m_{s}^{2}}
$$

It is then easy to verify that the scattering amplitude $T^{(-1)}+T^{(0)}+T^{(1)}$ is $\mu$-independent up to terms of order $Q^{2}$. Further, the effective range function is given at this order by

$$
k \cot \delta=-\frac{4 \pi}{m} \frac{1}{T^{(-1)}}\left[1-\frac{T^{(0)}}{T^{(-1)}}+\left(\frac{T^{(0)}}{T^{(-1)}}\right)^{2}-\frac{T^{(1)}}{T^{(-1)}}\right]+i k .
$$

One then obtains the following predictions for the ERE coefficients:

$$
\begin{aligned}
r^{\mathrm{NNLO}}= & \frac{1}{m_{l}}\left[\frac{3 \alpha_{l}-4}{\alpha_{l}}+\frac{2\left(\alpha_{l}-1\right)\left(3 \alpha_{l}-4\right) \alpha_{s}}{\alpha_{l}^{2} m_{s}} m_{l}\right. \\
& +\frac{\left(\alpha_{l}-1\right)\left(3 \alpha_{l}-4\right)\left(5 \alpha_{l}-3\right) \alpha_{s}^{2}+\left(2-\alpha_{l}\right) \alpha_{l}^{2}}{\alpha_{l}^{3} m_{s}^{2}} m_{l}^{2} \\
& \left.-\frac{4 \mu m_{l}\left(\alpha_{l}-1\right)\left(3 \alpha_{l}-4\right) \alpha_{s}^{3}\left(\pi m_{l}\left(3-5 \alpha_{l}\right)+4 \mu \alpha_{l}\right)}{\pi^{2} \alpha_{l}^{3} m_{s}^{3}}+\mathcal{O}\left(Q^{4}\right)\right], \\
v_{2}^{\mathrm{NNLO}=} & \frac{1}{m_{l}^{3}}\left[\frac{\alpha_{l}-2}{2 \alpha_{l}}+\frac{\left(\alpha_{l}\left(13 \alpha_{l}-36\right)+24\right) \alpha_{s}}{4 \alpha_{l}^{2} m_{s}} m_{l}\right. \\
& +\frac{\left(\alpha_{l}\left(\alpha_{l}\left(46 \alpha_{l}-159\right)+174\right)-60\right) \alpha_{s}^{2}-4\left(\alpha_{l}-2\right) \alpha_{l}^{2}}{4 \alpha_{l}^{3} m_{s}^{2}} m_{l}^{2} \\
& +\frac{\mu m_{l} \alpha_{s}^{3}\left(\pi m_{l}\left(\alpha_{l}\left(\alpha_{l}\left(46 \alpha_{l}-159\right)+174\right)-60\right)-2 \mu \alpha_{l}\left(\alpha_{l}\left(13 \alpha_{l}-36\right)+24\right)\right)}{\pi^{2} \alpha_{l}^{3} m_{s}^{3}}
\end{aligned}
$$




$$
\begin{aligned}
& \left.+\mathcal{O}\left(Q^{4}\right)\right] \\
v_{3}^{\mathrm{NNLO}}= & \frac{1}{m_{l}^{5}}\left[\frac{\left(\alpha_{l}-2\right)\left(3 \alpha_{l}-4\right) \alpha_{s}}{2 \alpha_{l}^{2} m_{s}} m_{l}+\frac{\left(3 \alpha_{l}-4\right)\left(\alpha_{l}\left(25 \alpha_{l}-68\right)+40\right) \alpha_{s}^{2}-4\left(\alpha_{l}-2\right) \alpha_{l}^{2}}{8 \alpha_{l}^{3} m_{s}^{2}} m_{l}^{2}\right. \\
& \left.+\frac{\mu m_{l}\left(3 \alpha_{l}-4\right) \alpha_{s}^{3}\left(\pi m_{l}\left(\alpha_{l}\left(25 \alpha_{l}-68\right)+40\right)-8 \mu\left(\alpha_{l}-2\right) \alpha_{l}\right)}{2 \pi^{2} \alpha_{l}^{3} m_{s}^{3}}+\mathcal{O}\left(Q^{4}\right)\right],(3.26)
\end{aligned}
$$

where $Q=\left\{m_{l}, \mu\right\}$. As expected, the first three terms in the "chiral" expansion of all ERE coefficients are reproduced correctly at NNLO. Notice further that the contributions beyond the order of accuracy of the calculation are explicitly renormalization-scale dependent, see section III for a general discussion. The above results reveal the meaning of the LETs in the present context. All $i$-th terms $\alpha_{x}^{(i)}$ in the "chiral" expansion of the coefficients in the ERE, $x=\left\{a, r, v_{2}, \ldots\right\}$ are correlated with each other due to the long-range interaction and its interplay with the short-range interaction in the underlying model. The knowledge of $\alpha_{x_{j}}^{(i)}$ for one particular $x_{j}$ is sufficient to predict $\alpha_{x_{k}}^{(i)}$ for all $k \neq j$. In an EFT, short-range physics is incorporated in a systematic way by taking into account contact interactions with an increasing number of derivatives. Matching the strengths of the corresponding LECs to the first $n$ terms in the "chiral" expansion of some of the ERE coefficients allows to correctly describe the "chiral" expansion of all ERE coefficients up to order $m_{l}^{n} / m_{s}^{n}$. It should be emphasized that at low energies and in the absence of external sources, the appearance of the above mentioned correlations is the only signature of the long-range interaction in the $2 \mathrm{~N}$ system.

\section{Weinberg-like approach with a finite cutoff}

An EFT formulation like the one described above which respects the manifest power counting at every stage of the calculation is not available in a general case of a long-range interaction which is strong enough to have to be treated non-perturbatively such as e. g. the one-pion exchange potential. Here, one lacks a regularization prescription for all divergent integrals resulting from iterations of the potential in the LS equation which would keep regularization artefacts small without, at the same time, introducing a new hard scale in the problem. In the context of pionful EFT for few-nucleon systems, the divergent integrals are usually dealt with by introducing an UV cutoff $\Lambda$. In order to keep regularization artefacts small, the cutoff, ideally, needs to be taken of the order $\Lambda \sim m_{s}$ or higher. Clearly, this spoils the manifest power counting for regularized loop contributions. ${ }^{3}$ We now consider the Weinberg-like formulation in which the effective potential, given by the long-range interaction and a series of contact terms, is iterated in the LS equation to all orders, see the work by Lepage [21] for a related discussion. This is visualized in Fig. 2. We carry out renormalization by literally following the steps outlined in Ref. [21] and summarized in Ref. [48] in the following way: "The theory is fully specified by the values of the bare constants ... once a suitable regularization procedure is chosen. In principle, the renormalization program is straightforward: one calculates quantities of physical interest in terms of the bare parameters at given, large value of (ultraviolet cutoff) $\Lambda$. Once a sufficient number of physical

\footnotetext{
${ }^{3}$ This, however, does not mean a breakdown of EFT since power counting is only required for renormalized scattering amplitude.
} 


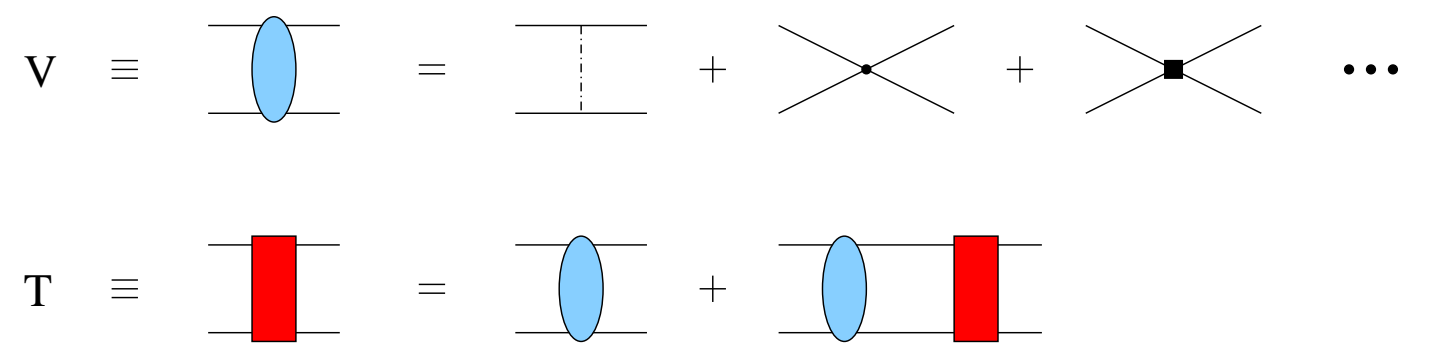

FIG. 2: Effective potential and scattering amplitude in the Weinberg-like approach. The dasheddotted line refers to the full long-range interaction. Solid dot and filled rectangle refer to the leading and subleading contact interactions, respectively. For remaining notation see Fig. 1 ,

quantities have been determined as functions of the bare parameters one inverts the result and expresses the bare parameters in terms of physical quantities, always working at some given, large value of $\Lambda$. Finally, one uses these expressions to eliminate the bare parameters in all other quantities of physical interest. Renormalizability guarantees that this operation at the same time also eliminates the cutoff." Notice that by iterating the truncated expansion for the effective potential in the LS equation one unavoidably generates higher-order contributions without being able to absorb all arising divergences into redefinition of the LECs present in the considered truncated potential. Thus, for the case at hand, cutoff dependence in observables is expected to be eliminated only up to the considered order in the EFT expansion. We further emphasize that expressing the bare parameters (i. e. LECs $C_{i}$ ) in terms of physical quantities is a non-trivial step due to a nonlinear dependence of the scattering amplitude on $C_{i}$. The resulting nonlinear equations may have no real solutions, especially when $\Lambda$ is chosen to be considerably larger than $m_{s}$. As pointed out in Ref. [21], "In fact, as nonlinearities develop for small $a$ 's, ${ }^{4}$ results often degrade, or, in more extreme cases, the theory may become unstable or untunable". The Wigner bound in pionless EFT [46] and the repulsive NN channels in the pionful case [38, 39] may serve as examples for such an untunable theory, see Ref. [28] for another example. In the following, we consider the low-energy theorems for our model and demonstrate explicitly that removing the cutoff from the scattering amplitude by taking the limit $\Lambda \rightarrow \infty$ is not compatible with the EFT framework even if such a limit exists and the theory does not become untunable.

To be specific, we consider the effective potential of the form

$$
V_{\mathrm{eff}}^{(1)}\left(p, p^{\prime}\right)=v_{l} F_{l}(p) F_{l}\left(p^{\prime}\right)+C_{0},
$$

where the superscript of $V_{\text {eff }}$ refers to the number of contact terms included. The off-shell T-matrix $T^{(1)}\left(p, p^{\prime} ; k\right)$ can be easily calculated by solving the $2 \times 2$ matrix equation

$$
t(k)=v_{\text {eff }}+v_{\text {eff }} \mathcal{G}(k) t(k)
$$

where we have defined

$$
V_{\mathrm{eff}}^{(1)}\left(p, p^{\prime}\right)=\gamma^{T}(p) v_{\mathrm{eff}} \gamma\left(p^{\prime}\right), \quad T^{(1)}\left(p, p^{\prime}, k\right)=\gamma^{T}(p) t(k) \gamma\left(p^{\prime}\right),
$$

\footnotetext{
${ }^{4}$ In this work, $a$ refers to a coordinate-space cutoff, $a \sim \Lambda^{-1}$.
} 
with

$$
v_{\text {eff }} \equiv\left(\begin{array}{cc}
v_{l} & 0 \\
0 & C_{0}
\end{array}\right), \quad \gamma(p) \equiv\left(\begin{array}{c}
F_{l}(p) \\
1
\end{array}\right), \quad \mathcal{G}(k) \equiv\left(\begin{array}{cc}
I_{l}(k) & I_{l 1}^{\mathrm{reg}}(k) \\
I_{l 1}^{\mathrm{reg}}(k) & I_{1}^{\mathrm{reg}}(k)
\end{array}\right) .
$$

The integrals entering $\mathcal{G}(k)$ are given by

$$
\begin{aligned}
I_{l}(k)= & 4 \pi m \int_{0}^{\infty} \frac{l^{2} d l}{(2 \pi)^{3}} \frac{l^{2}+m_{s}^{2}}{\left[k^{2}-l^{2}+i \epsilon\right]\left[l^{2}+m_{l}^{2}\right]^{2}} \\
= & \frac{m\left(-2 i k m_{l}+m_{l}^{2}+m_{s}^{2}\right)}{8 \pi m_{l}\left(k+i m_{l}\right)^{2}}, \\
I_{l 1}^{\mathrm{reg}}(k)= & 4 \pi m \int_{0}^{\Lambda} \frac{l^{2} d l}{(2 \pi)^{3}} \frac{\sqrt{l^{2}+m_{s}^{2}}}{\left[k^{2}-l^{2}+i \epsilon\right]\left[l^{2}+m_{l}^{2}\right]} \\
= & \frac{m}{2 \pi^{2}}\left(k \frac{\sqrt{k^{2}+m_{s}^{2}}}{k^{2}+m_{l}^{2}} \ln \left(\frac{k+\sqrt{k^{2}+m_{s}^{2}}}{m_{s}}\right)-\frac{m_{l} \sqrt{m_{s}^{2}-m_{l}^{2}}}{k^{2}+m_{l}^{2}} \operatorname{arccot}\left(\frac{m_{l}}{\sqrt{m_{s}^{2}-m_{l}^{2}}}\right)\right. \\
& \left.+\ln \left(\frac{m_{s}}{2 \Lambda}\right)-\frac{i \pi k \sqrt{k^{2}+m_{s}^{2}}}{2\left(k^{2}+m_{l}^{2}\right)}\right)+\mathcal{O}\left(\Lambda^{-1}\right),
\end{aligned}
$$

and the integral $I_{1}^{\text {reg }}(k)$ is defined in Eq. (3.19). Here and in what follows, we keep the cutoff $\Lambda$ at least of the order $\Lambda \sim m_{s}$. We will, therefore, omit the finite cutoff artefacts in order to keep the presentation simple, i. e. we neglect the $\mathcal{O}\left(\Lambda^{-1}\right)$-terms in Eqs. (3.19) and (3.31). The reader can easily verify that taking into account finite cutoff artefacts (i.e. terms with negative powers of $\Lambda$ ) in the expressions for regularized loop integrals does not alter the conclusions of this work. With the above definitions, the LS equation (3.28) can be easily solved leading to a somewhat lengthy expressions for the on-shell T-matrix $T^{(1)}(k, k ; k)$ which can be used to extract the coefficients in the ERE. One obtains for the scattering length

$$
a^{(1)}=\frac{\pi m_{s}\left\{C_{0} m\left[2 \alpha_{l}\left(m_{s}\left(\Lambda-\mathrm{s} m_{l}\right)+2 m_{l}^{2} \ln \left(m_{s} / 2 \Lambda\right)\right)+\pi m_{l} m_{s}\right]+4 \pi^{2} \alpha_{l} m_{s}\right\}}{m_{l}\left\{2 \pi m_{s}^{2}\left(C_{0} m \Lambda+2 \pi^{2}\right)-C_{0} m m_{l} \alpha_{l}\left[\mathrm{~s} m_{s}-2 m_{l} \ln \left(m_{s} / 2 \Lambda\right)\right]^{2}\right\}},
$$

where we have introduced

$$
s \equiv 2 \frac{\sqrt{m_{s}^{2}-m_{l}^{2}}}{m_{s}} \operatorname{arccot}\left(\frac{m_{l}}{\sqrt{m_{s}^{2}-m_{l}^{2}}}\right) .
$$

One can, in principle, determine the LEC $C_{0}$ by expanding $a^{(1)}$ in powers of $m_{l}$ and matching the second term in this expansion to $\alpha_{a}^{(2)}$ as we did in the case of the KSW-approach. However, in practice, "chiral" expansion of the coefficients in the ERE is not available. We, therefore, determine $C_{0}$ for a given value of the cutoff $\Lambda$, i.e. $C_{0}(\Lambda)$, by matching $a^{(1)}$ to the full expression of the scattering length resulting in our model

$$
a_{\text {underlying }}=\frac{m_{l}\left(2 \alpha_{l}-1\right) \alpha_{s}-\alpha_{l} m_{s}}{m_{l}\left(m_{l} \alpha_{l} \alpha_{s}-m_{s}\right)},
$$

which we regard as a synthetic data. This leads to the following result for $C_{0}(\Lambda)$ :

$$
C_{0}(\Lambda)=4 \pi^{3}\left(\alpha_{l}-1\right)^{2} m_{s}^{2} \alpha_{s}\left\{m m_{s}\left[m_{s}\left(\pi-\mathrm{s} \alpha_{l}\right)+2 m_{l} \alpha_{l} \ln \frac{m_{s}}{2 \Lambda}\right]^{2}\right.
$$




$$
\begin{aligned}
& -m \alpha_{s}\left[m_{s}^{2}\left(\alpha_{l}\left((\pi-\mathrm{s}) m_{l}\left(-2 \mathrm{~s} \alpha_{l}+\mathrm{s}+\pi\right)+2 \pi \Lambda\left(\alpha_{l}-2\right)\right)+2 \pi \Lambda\right)\right. \\
& \left.\left.+4 m_{l}^{2} \alpha_{l} m_{s}\left((\pi-2 \mathrm{~s}) \alpha_{l}+\mathrm{s}\right) \ln \frac{m_{s}}{2 \Lambda}+4 m_{l}^{3} \alpha_{l}\left(2 \alpha_{l}-1\right)\left(\ln \frac{m_{s}}{2 \Lambda}\right)^{2}\right]\right\}^{-1} .
\end{aligned}
$$

Having determined the LEC $C_{0}(\Lambda)$, we are now in the position to verify the low-energy theorems by making predictions for the effective range and shape coefficients. A straightforward calculation yields the following result for renormalized expressions for $r^{(1)}$ and $v_{2}^{(1)}$ :

$$
\begin{aligned}
& r^{(1)}=\frac{1}{m_{l}}\left[\frac{3 \alpha_{l}-4}{\alpha_{l}}+\frac{2\left(\alpha_{l}-1\right)\left(3 \alpha_{l}-4\right) \alpha_{s}}{\alpha_{l}^{2} m_{s}} m_{l}+\left(\frac{4\left(\alpha_{l}-2\right) \alpha_{s}}{\pi \alpha_{l} m_{s}^{2}}\left(\ln \frac{m_{s}}{2 \Lambda}+1\right)\right.\right. \\
& \left.\left.+\frac{\left(\alpha_{l}-1\right)\left(3 \alpha_{l}-4\right)\left(5 \alpha_{l}-3\right) \alpha_{s}^{2}+\left(2-\alpha_{l}\right) \alpha_{l}^{2}}{\alpha_{l}^{3} m_{s}^{2}}\right) m_{l}^{2}+\mathcal{O}\left(m_{l}^{3}\right)\right], \\
& v_{2}^{(1)}=\frac{1}{m_{l}^{3}}\left[\frac{\alpha_{l}-2}{2 \alpha_{l}}+\frac{\left(\alpha_{l}\left(13 \alpha_{l}-36\right)+24\right) \alpha_{s}}{4 \alpha_{l}^{2} m_{s}} m_{l}+\left(\frac{\left(\alpha_{l}-2\right)\left(5 \alpha_{l}-6\right) \alpha_{l}^{2} \alpha_{s}}{\pi\left(\alpha_{l}-1\right) \alpha_{l}^{3} m_{s}^{2}}\left(\ln \frac{m_{s}}{2 \Lambda}+1\right)\right.\right. \\
& \left.\left.+\frac{\left(\alpha_{l}\left(\alpha_{l}\left(46 \alpha_{l}-159\right)+174\right)-60\right) \alpha_{s}^{2}-4\left(\alpha_{l}-2\right) \alpha_{l}^{2}}{4 \alpha_{l}^{3} m_{s}^{2}}\right) m_{l}^{2}+\mathcal{O}\left(m_{l}^{3}\right)\right], \\
& v_{3}^{(1)}=\frac{1}{m_{l}^{5}}\left[\frac{\left(\alpha_{l}-2\right)\left(3 \alpha_{l}-4\right) \alpha_{s}}{2 \alpha_{l}^{2} m_{s}} m_{l}+\left(\frac{2\left(\alpha_{l}-2\right)\left(2 \alpha_{l}-3\right) \alpha_{s}}{\pi\left(\alpha_{l}-1\right) \alpha_{l} m_{s}^{2}}\left(\ln \frac{m_{s}}{2 \Lambda}+1\right)\right.\right. \\
& \left.\left.+\frac{\left(3 \alpha_{l}-4\right)\left(\alpha_{l}\left(25 \alpha_{l}-68\right)+40\right) \alpha_{s}^{2}-4\left(\alpha_{l}-2\right) \alpha_{l}^{2}}{8 \alpha_{l}^{3} m_{s}^{2}}\right) m_{l}^{2}+\mathcal{O}\left(m_{l}^{3}\right)\right] \text {. }
\end{aligned}
$$

Again, not surprisingly, one observes that the subleading terms in the "chiral" expansion of the ERE coefficients are correctly reproduced. In fact, any quantum-mechanically welldefined short-range interaction accompanied with the underlying long-range force would do equally good job in describing correlations between $\alpha_{x}^{(1)}$. While all coefficients $\alpha_{x}^{(1)}$ have to be reproduced correctly at this order once the short-range parameter entering the effective potential is appropriately tuned (as guaranteed by the analytic structure of the scattering amplitude), there is no restriction regarding higher-order terms in the "chiral" expansion. ${ }^{5}$ Indeed, one observes that the coefficients $\alpha_{r, v_{i}}^{(2)}$ in Eq. (3.36) deviate from their correct values given in Eqs. (3.8)-(3.10). Moreover, since the included LEC is insufficient to absorb all divergencies arising from iterations of the LS equation, nothing prevents the appearance of positive powers or logarithms of the cutoff $\Lambda$ in the expressions for $\alpha_{r, v_{i}}^{(n)}$ with $n \geq 2{ }^{6}$ The results in Eq. (3.36) show that this is indeed the case. The dependence on $\Lambda$ occurs,

\footnotetext{
${ }^{5}$ A careful reader may realize that also the sub-subleading coefficients in the "chiral" expansion of $r, v_{2}$ and $v_{3}$ are correctly reproduced once the cutoff is tuned to the value $\Lambda=e m_{s} / 2$. This, in fact, also holds true for higher coefficients in the ERE and can be traced back to the fact that in the case at hand, the cutoff $\Lambda$ itself may be considered as an additional short-range "counterterm" provided one allows for a fine tuning of $\Lambda$. The resulting expressions are completely equivalent to the next-higher-order calculation in the Weinberg-like approach and provide explicit evidence for the validity of low-energy theorems in that case.

${ }^{6}$ The appearance of only logarithmic dependence on $\Lambda$ in Eq. (3.36) is specific to the form of the long-range interaction and the order in the EFT expansion. We have verified that positive powers of $\Lambda$ occur in the expressions for $\alpha_{x}^{(3)}$ when one includes the subleading contact interaction in the effective potential.
} 
however, only in contributions beyond the accuracy of calculation and, obviously, does not affect the predictive power of the EFT provided the cutoff is chosen to be of the order of the characteristic hard scale in the problem, $\Lambda \sim m_{s}$. Taking values $\Lambda \gg m_{s}$ artificially enhances certain higher-order contributions in the "chiral" expansion of the ERE coefficients spoiling the predictive power of the theory.

The appearance of positive powers of $\Lambda$ and/or logarithmic terms in the predicted "chiral" expansion of the effective range and the shape parameters in Eq. (3.36) may give the wrong impression that no finite limit exists for $r^{(1)}(\Lambda)$ and $v_{i}^{(1)}(\Lambda)$ as $\Lambda \rightarrow \infty$. In fact, taking the limit $\Lambda \rightarrow \infty$ does not commute with the Taylor expansion of the ERE coefficients in powers of $m_{l}$. It is easy to see, that all coefficients in the ERE as well as the on-shell T-matrix approach a finite limit as $\Lambda \rightarrow \infty$. Substituting the value for $C_{0}(\Lambda)$ from Eq. (3.35) into the solution of the LS equation (3.28) and taking the limit $\Lambda \rightarrow \infty$ one obtains the following cutoff-independent result for the inverse amplitude:

$$
\begin{aligned}
\left(T_{\text {peratized }}^{(1)}\right)^{-1}= & i \frac{k m}{4 \pi}-\frac{m}{8 \pi m_{l}^{3}\left(k^{2}+m_{s}^{2}\right)\left(\alpha_{l} m_{s}+m_{l}\left(1-2 \alpha_{l}\right) \alpha_{s}\right)}\left(2 m_{l}^{4} m_{s}^{2}\left(m_{s}-m_{l} \alpha_{l} \alpha_{s}\right)\right. \\
& +k^{2} m_{l}^{2}\left(\left(4-3 \alpha_{l}\right) m_{s}^{3}+m_{l}^{2} \alpha_{l} m_{s}+m_{l} \alpha_{s}\left(\left(2 \alpha_{l}-3\right) m_{s}^{2}+m_{l}^{2}\left(1-2 \alpha_{l}\right)\right)\right) \\
& \left.+k^{4}\left(-\alpha_{l} m_{s}\left(m_{l}^{2}+m_{s}^{2}\right)-m_{l} \alpha_{s}\left(m_{l}^{2}\left(1-2 \alpha_{l}\right)+m_{s}^{2}\right)+2 m_{s}^{3}\right)\right)
\end{aligned}
$$

The above procedure is very much in spirit of the so-called peratization, the technique introduced by Feinberg and Pais [49, 50], see also [51], to evaluate higher-order corrections to S-matrix in non-renormalizable field theories. The essential idea of this method consists in the resummation of the most divergent contributions to the Born series. In the late sixties of the last century, this technique was widely used in potential scattering as an attempt to generate approximations to the scattering length for different classes of singular potentials. In some cases where the exact solution to the Schrödinger equation with a given singular potential is known, peratization was indeed shown to provide reasonable approximations to the scattering length while in other cases this approach fails completely, see [52] for a comprehensive review article.

The cutoff-removed results for the ERE coefficients can be read off from Eq. (3.37):

$$
\begin{aligned}
r_{\text {peratized }}^{(1)}= & \frac{m_{l}^{3} \alpha_{s}+m_{l}^{2}\left(\alpha_{l}-2\right) m_{s}+m_{l}\left(2 \alpha_{l}-3\right) m_{s}^{2} \alpha_{s}+\left(4-3 \alpha_{l}\right) m_{s}^{3}}{m_{l} m_{s}^{2}\left(m_{l}\left(2 \alpha_{l}-1\right) \alpha_{s}-\alpha_{l} m_{s}\right)} \\
= & \frac{1}{m_{l}}\left[\frac{3 \alpha_{l}-4}{\alpha_{l}}+\frac{4\left(\alpha_{l}-1\right)^{2} \alpha_{s}}{\alpha_{l}^{2} m_{s}} m_{l}\right. \\
& \left.+\frac{\alpha_{l}^{3}\left(8 \alpha_{s}^{2}-1\right)+\alpha_{l}^{2}\left(2-20 \alpha_{s}^{2}\right)+16 \alpha_{l} \alpha_{s}^{2}-4 \alpha_{s}^{2}}{\alpha_{l}^{3} m_{s}^{2}} m_{l}^{2}+\mathcal{O}\left(m_{l}^{3}\right)\right] \\
\left(v_{2}^{(1)}\right)_{\text {peratized }}= & -\frac{\left(m_{l}^{2}-m_{s}^{2}\right)^{2}\left(\left(\alpha_{l}-2\right) m_{s}+m_{l} \alpha_{s}\right)}{2 m_{l}^{3} m_{s}^{4}\left(m_{l}\left(2 \alpha_{l}-1\right) \alpha_{s}-\alpha_{l} m_{s}\right)} \\
= & \frac{1}{m_{l}^{3}}\left[\frac{\alpha_{l}-2}{2 \alpha_{l}}+\frac{\left(\alpha_{l}-1\right)^{2} \alpha_{s}}{\alpha_{l}^{2} m_{s}}+\frac{\alpha_{l}^{3}\left(2 \alpha_{s}^{2}-1\right)+\alpha_{l}^{2}\left(2-5 \alpha_{s}^{2}\right)+4 \alpha_{l} \alpha_{s}^{2}-\alpha_{s}^{2}}{\alpha_{l}^{3} m_{s}^{2}} m_{l}^{2}\right. \\
& \left.+\mathcal{O}\left(m_{l}^{3}\right)\right], \\
& \frac{\left(m_{l}^{2}-m_{s}^{2}\right)^{2}\left(\left(\alpha_{l}-2\right) m_{s}+m_{l} \alpha_{s}\right)}{2 m_{l}^{3} m_{s}^{6}\left(m_{l}\left(2 \alpha_{l}-1\right) \alpha_{s}-\alpha_{l} m_{s}\right)}
\end{aligned}
$$




$$
=\frac{1}{m_{l}^{5}}\left[-\frac{\alpha_{l}-2}{2 \alpha_{l} m_{s}^{2}} m_{l}^{2}+\mathcal{O}\left(m_{l}^{3}\right)\right] .
$$

One observes that the results after removing the cutoff fail to reproduce the low-energy theorems by yielding wrong values for $\alpha_{r}^{(1)}$ and $\alpha_{v_{i}}^{(1)}$ (notice that, per construction, the scattering length corresponding to $T_{\text {peratized }}^{(1)}$ exactly matches $a_{\text {underlying }}$ ). Note that we could allow for a stronger fine-tuning in our model to make both the long- and short-range interactions nonperturbative at $k \sim m_{l}$ (as it probably happens in the realistic case of NN scattering). The breakdown of LETs in the "peratized" expressions would then imply that the coefficients in the ERE are completely uncorrelated with each other, that is, the predictive power of such an approach is the same as in the theory with only short-range interactions (i. e. "pionless" theory).

The breakdown of LETs in the "peratized" approach can be traced back to spurious $\Lambda$ dependent contributions in the T-matrix which are irrelevant (at the order of calculations) in the regime $\Lambda \sim m_{s}$ but become numerically dominant if $\Lambda \gg m_{s}$. In general, such spurious terms involve positive powers of $\Lambda$ which, as $\Lambda$ gets increased beyond the hard scale $m_{s}$, become, at some point, comparable in size with the lower-order terms. For example, as already mentioned before, terms linear in $\Lambda$ will show up in the renormalized expressions for $\alpha_{x}^{(3)}$ at next-higher order. Low-energy theorems will then break down as the cutoff will approach the scale $\Lambda \sim m_{s}^{2} / m_{l}$. The unavoidable appearance of ever higher power-law divergences when going to higher orders in the EFT expansion implies that the cutoff should not be increased beyond the hard scale in the problem, which leads to the optimal choice $\Lambda \sim m_{s}$.

\section{SUMMARY AND CONCLUSIONS}

We discussed some conceptual aspects of renormalization in the context of effective field theories for the two-nucleon system. First, we considered renormalization scheme dependence of the scattering amplitude in the KSW and Weinberg's approaches. Renormalization scale dependence is present explicitly in the loop contributions and implicitly due to the running of the coupling constants. These two types of dependence cancel exactly in the full amplitude. Contrary to widespread belief, we showed that renormalization scheme independence of the amplitude in pionless EFT based on the KSW framework is only achievable up to the order to which the calculations are performed. The residual renormalizationscheme dependence arises from the running coupling constants. On the other hand, in the Weinberg's approach the residual renormalization scale dependence is generated by loop contributions. From this point of view, the KSW framework does not offer any conceptual advantage over the Weinberg's approach. If one approach is conceptually inconsistent, then so is the other. In fact both are conceptually as consistent as perturbative QCD. Clearly, the crucial point is to choose the appropriate renormalization condition.

Secondly, we considered the cutoff version of pionless theory for NN scattering in the ${ }^{1} S_{0}$ partial wave up to next-to-leading order. We expressed the scattering amplitude in terms of renormalized coupling constants and explored the consequences of taking the cutoff $\Lambda$ very large, i.e. much larger than the hard scale in the problem. Making use of the loop expansion for the scattering amplitude, we observed that the contributions which diverge in the limit $\Lambda \rightarrow \infty$, instead of being absorbed into redefinition of higher-order coupling constants (or, equivalently, being subtracted), start playing a dominant role as $\Lambda$ is increased significantly 
beyond the pertinent hard scale. One, therefore, completely looses the power counting (at the level of the amplitude) on which the EFT is based. On the other hand, if $\Lambda$ is chosen of the order of the hard scale, violation of the power counting by terms proportional to $\Lambda$ appears to be beyond the accuracy of the calculation.

To further explore the role of the cutoff we constructed a toy-model for pionful EFT. Specifically, we developed an effective theory for an exactly solvable quantum mechanical problem with long- and short-range interactions of a separable type. We revealed the meaning of low-energy theorems in this model using the KSW-like framework with subtractive renormalization and demonstrated their validity in the Weinberg-like approach with a finite cutoff $\Lambda$ as long as it is chosen of the order of short-range scale. Taking the limit $\Lambda \rightarrow \infty$ while keeping the scattering length at its correct value yields a finite result for the amplitude but violates the low-energy theorems. This procedure is, therefore, not compatible with the EFT framework. It is much more in spirit of peratization [49, 50, 51, 52] than renormalization as it is understood in the context of EFT. Contrary to popular opinion, the considered example demonstrates that the existence of a finite limit of the amplitude as $\Lambda \rightarrow \infty$ under requirement, that certain low-energy observables such as e. g. the scattering length are kept at their physical values, is not yet sufficient for a proper renormalization in the context of chiral EFT (neither is it necessary, see e.g. [21, 23]). We argue that $\Lambda$ should not be increased (considerably) beyond the short-range scale in the problem in EFT calculations of that kind.

\section{Acknowledgments}

We would like to thank Dalibor Djukanovic, Ulf-G. Meißner, Daniel Phillips and Manuel Pavón Valderrama for useful comments on the manuscript. The work of E.E. was supported by funds provided by the Helmholtz Association to the young investigator group "Few-Nucleon Systems in Chiral Effective Field Theory" (grant VH-NG-222) and to the virtual institute "Spin and strong QCD" (VH-VI-231), by the DFG (SFB/TR 16 "Subnuclear Structure of Matter") and by the EU HadronPhysics2 project "Study of strongly interacting matter". J.G. acknowledges the support of the Deutsche Forschungsgemeinschaft (SFB 443) and Georgian National Foundation grant GNSF/ST08/4-400.

[1] S. Weinberg, Phys. Lett. B 251, 288 (1990).

[2] S. Weinberg, Nucl. Phys. B363, 3 (1991).

[3] C. Ordonez and U. van Kolck, Phys. Lett. B 291, 459 (1992).

[4] P. F. Bedaque and U. van Kolck, Ann. Rev. Nucl. Part. Sci. 52, 339 (2002).

[5] E. Epelbaum, Prog. Part. Nucl. Phys. 57, 654 (2006).

[6] E. Epelbaum, H. W. Hammer and U.-G. Meißner, arXiv:0811.1338 [nucl-th], to appear in Rev. Mod. Phys..

[7] D. B. Kaplan, M. J. Savage, and M. B. Wise, Nucl. Phys. B478, 629 (1996).

[8] S. R. Beane, P. F. Bedaque, M. J. Savage and U. van Kolck, Nucl. Phys. A 700, 377 (2002).

[9] D. B. Kaplan, M. J. Savage, and M. B. Wise, Phys. Lett. B 424, 390 (1998).

[10] D. B. Kaplan, M. J. Savage, and M. B. Wise, Nucl. Phys. B534, 329 (1998).

[11] M. J. Savage, arXiv:nucl-th/9804034. 
[12] S. Fleming, T. Mehen, and I. W. Stewart, Nucl. Phys. A677, 313 (2000).

[13] S. R. Beane, D. B. Kaplan and A. Vuorinen, arXiv:0812.3938 [nucl-th].

[14] J. Gegelia, arXiv:nucl-th/9806028.

[15] J. Gegelia, Phys. Lett. B 463, 133 (1999).

[16] T. D. Cohen and J. M. Hansen, Phys. Rev. C 59, 13 (1999).

[17] T. D. Cohen and J. M. Hansen, Phys. Rev. C 59, 3047 (1999).

[18] T. D. Cohen and J. M. Hansen, arXiv:nucl-th/9908049.

[19] D. R. Entem and R. Machleidt, Phys. Rev. C 68, 041001 (2003).

[20] E. Epelbaum, W. Glöckle and U.-G. Meißner, Nucl. Phys. A 747, 362 (2005).

[21] G.P. Lepage, arXiv:nucl-th/9706029.

[22] G. P. Lepage, Conference summary, Prepared for INT Workshop on Nuclear Physics with Effective Field Theory, Seattle, Washington, 25-26 Feb 1999.

[23] G.P. Lepage, How to renormalize the Schrödinger equation, talk given at the INT program Effective Field Theories and Effective Interactions, INT, Seattle, USA, June 25-August 2, 2000 .

[24] J. Gegelia, Phys. Lett. B 429, 227 (1998).

[25] J. Gegelia, J. Phys. G 25, 1681 (1999).

[26] T. S. Park, K. Kubodera, D. P. Min, and M. Rho, Nucl. Phys. A646, 83 (1999).

[27] J. Gegelia and S. Scherer, Int. J. Mod. Phys. A 21, 1079 (2006).

[28] E. Epelbaum and U. -G. Meißner, arXiv:nucl-th/0609037.

[29] A. Nogga, R. G. E. Timmermans, and U. van Kolck, Phys. Rev. C 72, 054006 (2005).

[30] M. C. Birse and J. A. McGovern, Phys. Rev. C 70, 054002 (2004).

[31] T. Barford and M. C. Birse, Phys. Rev. C 67, 064006 (2003).

[32] M. C. Birse, Phys. Rev. C 74, 014003 (2006).

[33] T. Frederico, V. S. Timoteo and L. Tomio, Nucl. Phys. A 653, 209 (1999).

[34] M. Pavon Valderrama and E. Ruiz Arriola, Phys. Lett. B 580, 149 (2004).

[35] M. Pavon Valderrama and E. Ruiz Arriola, Phys. Rev. C 70, 044006 (2004).

[36] V. S. Timoteo, T. Frederico, A. Delfino and L. Tomio, Phys. Lett. B 621, 109 (2005).

[37] M. Pavon Valderrama and E. Ruiz Arriola, Phys. Rev. C 72, 054002 (2005).

[38] M. Pavon Valderrama and E. R. Arriola, Phys. Rev. C 74, 054001 (2006).

[39] M. Pavon Valderrama and E. Ruiz Arriola, Phys. Rev. C 74, 064004 (2006) [Erratum-ibid. C 75, 059905 (2007)].

[40] R. Higa, M. Pavon Valderrama and E. Ruiz Arriola, Phys. Rev. C 77, 034003 (2008).

[41] D. R. Entem, E. Ruiz Arriola, M. Pavon Valderrama and R. Machleidt, Phys. Rev. C 77, 044006 (2008).

[42] B. Long and U. van Kolck, Annals Phys. 323, 1304 (2008).

[43] C. J. Yang, C. Elster and D. R. Phillips, Phys. Rev. C 77, 014002 (2008).

[44] M. P. Valderrama and E. R. Arriola, arXiv:0809.3186 [nucl-th].

[45] C. J. Yang, C. Elster and D. R. Phillips, arXiv:0901.2663 [nucl-th].

[46] D. R. Phillips, S. R. Beane and T. D. Cohen, Annals Phys. 263, 255 (1998).

[47] S. R. Beane, T. D. Cohen and D. R. Phillips, Nucl. Phys. A 632, 445 (1998).

[48] J. Gasser and H. Leutwyler, Phys. Rept. 87, 77 (1982).

[49] G. Feinberg and A. Pais, Phys. Rev. 131, 2724 (1963).

[50] G. Feinberg and A. Pais, Phys. Rev. 133, B477 (1964).

[51] W. Guttinger, R. Penzl and E. Pfaffelhuber, Nuovo Cim. 35, 1247 (1965).

[52] W. Frank, D. J. Land and R. M. Spector, Rev. Mod. Phys. 43, 36 (1971). 